\title{
Conserved microsatellite markers of high cross-species utility for flying, ground and tree squirrels
}

\author{
Salisa Jumpa $\cdot$ Deborah A. Dawson • \\ Gavin J. Horsburgh · Catherine Walton
}

Received: 23 January 2015/ Accepted: 14 February 2015/Published online: 26 February 2015

(C) The Author(s) 2015. This article is published with open access at Springerlink.com

\begin{abstract}
Many squirrel species around the world are threatened by forest loss and fragmentation. To facilitate studies of squirrel biodiversity, particularly of flying squirrels in Southeast Asia, we identified Hylopetes, Menetes, Glaucomys and Sciurus squirrel microsatellite sequences with homologs in a second squirrel species (Spermophilus tridecemlineatus), designed 40 consensus markers and tested three squirrel species. When tested in four individuals per species, 26 markers were variable in Hylopetes phayrei, 25 markers in H. lepidus and 25 markers in Menetes berdmorei. Eleven markers were selected from 14 that were polymorphic in all three species. Cross-species utility was confirmed for these 11 markers in seven additional squirrel species, including: the flying squirrels H. phayrei, H. lepidus, H. spadiceus and Petaurista petaurista; a ground squirrel, $M$. berdmorei; and the tree squirrels, Callosciurus caniceps and $C$. finlaysoni. The other markers that were variable in one or multiple species are also useful for those specific species.
\end{abstract}

Keywords Enhanced cross-species utility $\cdot$ Sciuridae . Simple tandem repeat (STR) $\cdot$ Squirrel $\cdot$ Thailand

Electronic supplementary material The online version of this article (doi:10.1007/s12686-015-0439-1) contains supplementary material, which is available to authorized users.

S. Jumpa $\cdot$ C. Walton $(\bowtie)$

Faculty of Life Sciences, University of Manchester, Michael Smith Building, Oxford Road, Manchester M13 9PT, UK

e-mail: Catherine.Walton@manchester.ac.uk

S. Jumpa · D. A. Dawson - G. J. Horsburgh

NERC Biomolecular Analysis Facility, Department of Animal and Plant Sciences, University of Sheffield, Western Bank, Sheffield, South Yorkshire S10 2TN, UK
Many forest taxa, including squirrels, are becoming increasingly endangered due to the effects of forest loss and fragmentation (Sodhi et al. 2010). This is particularly true in Southeast Asia that has the highest rates of forest loss worldwide (Sodhi et al. 2004). In Southeast Asia, squirrel species vary in IUCN status from Critically Endangered (e.g. Biswamoyopterus biswasi (the Namdapha Flying Squirrel) in northeast India) to Least Concern (http://www.iucnredlist. org/details/2816/0). However the risks are certainly underestimated as true species diversity remains unknown and many taxa are classified by the IUCN as Data Deficient. It is important to note that even though some species are listed as Least Concern they actually face significant threats. For example, two of our study species: Hylopetes lepidus (the Grey-cheeked Flying Squirrel); and Hylopetes phayrei (Phayre's Flying Squirrel), are hunted extensively and sold in food markets throughout Thailand (SJ personal observation), despite being officially protected under the Preservation and Protection of Wildlife Act of B.E. 2535 (1992) (http://www.forest.go.th). Here we developed conserved microsatellite markers to facilitate conservation genetics studies in a broad range of squirrel taxa but particularly flying squirrels of the genus Hylopetes from Indochina.

Genomic DNA was extracted from ear clips using a phenol-chloroform extraction method. Microsatellite-enriched genomic libraries were constructed separately following Armour et al. (1994) using one adult female from each of three species: the flying squirrels: $H$. phayrei and H. lepidus; and the Indochinese Ground Squirrel, Menetes berdmorei, all sampled in Thailand. From these we generated 89,85 and 91 unique microsatellite sequences (EMBL accession numbers LN650709-650973), respectively. Microsatellite sequences were available from GenBank for five other squirrel species: Glaucomys sabrinus, G. volans, Sciurus lis, S. niger and S. vulgaris. 


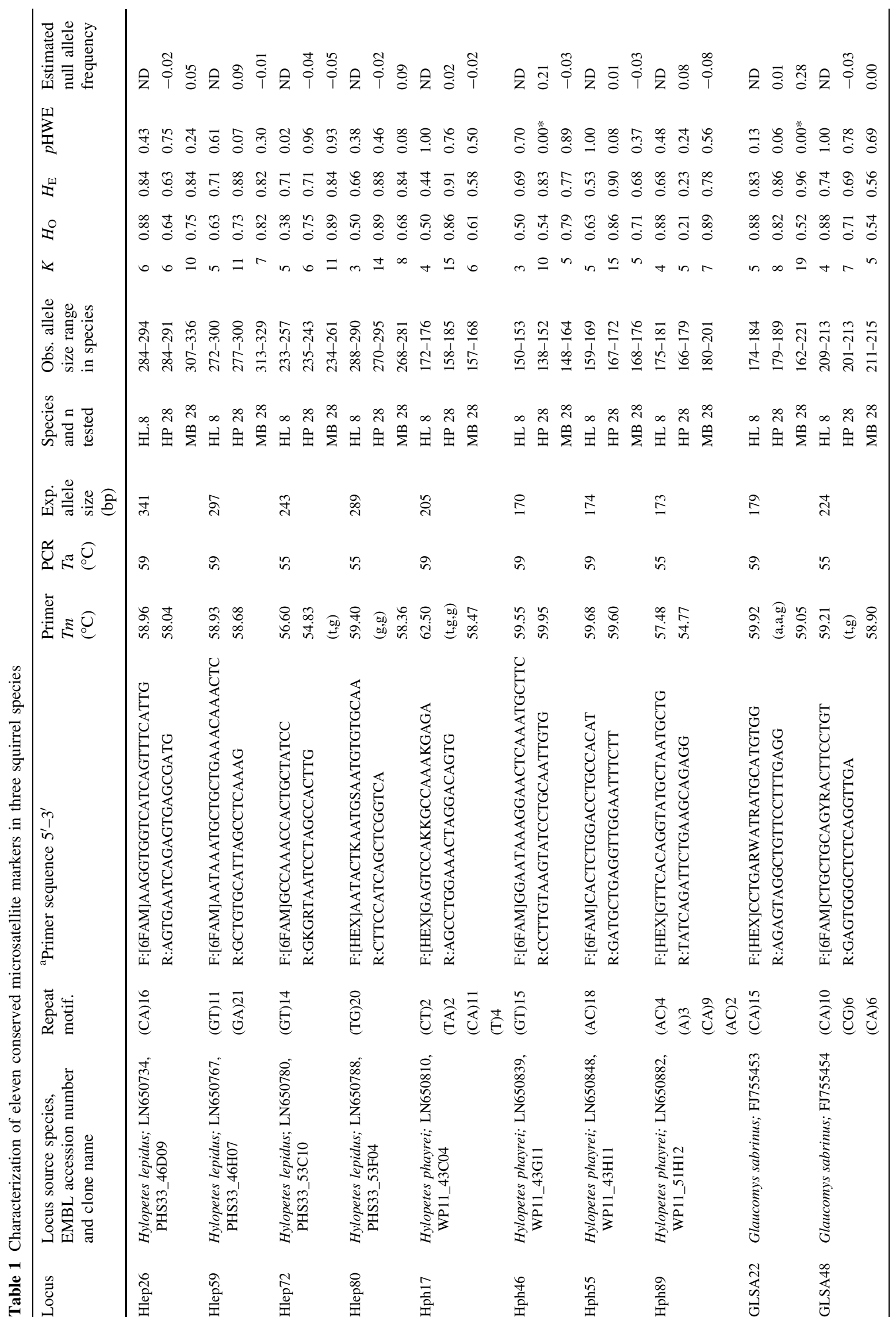


We followed the approach of Dawson et al. (2010) to identify conserved microsatellite sequences and create markers of high cross-species utility. We created a consensus sequence by aligning the newly isolated squirrel microsatellite sequences and/or other online squirrel sequences (Hylopetes/Menetes/Glaucomys/Sciurus) against their homologue in the thirteen-lined ground squirrel genome (Spermophilus tridecemlineatus; http://www.ensembl. org/Spermophilus_tridecemlineatus/index.html). Primer sets were designed for 40 microsatellite loci based on these consensus sequences using PRIMER3 v0.4.0 (avoiding bases mismatching between species, when possible). Each primer set matched S. tridecemlineatus and one of the other eight squirrel species cited above (the "source" species) with a maximum of three degenerate bases per primer (three per primer set) and a maximum of one base mismatching $S$. tridecemlineatus. The optimal difference between the forward and reverse primer melting temperatures was set to $0.5{ }^{\circ} \mathrm{C}$ (maximum $4{ }^{\circ} \mathrm{C}$; Table 1 ).

The 40 conserved microsatellite markers were tested in our primary study species $H$. phayrei, H. lepidus and $M$. berdmorei (using four individuals per species). Twenty-six markers were variable in $H$. phayrei, 25 markers in H. lepidus and 25 markers in M. berdmorei (Supplementary Table 2). Fourteen loci amplified and were polymorphic in all three species (Supplementary Table 2). Primer sets designed from comparisons of the thirteen-lined ground squirrel with the flying squirrels (Hylopetes) amplified in more species than those designed from comparisons between the two ground squirrel species, $M$. berdmorei and $S$. tridecemlineatus (Supplementary Tables 1 and 2). This is likely due to the greater phylogenetic distance between Hylopetes/Glaucomys/Sciurus genera and S. tridecemlineatus than between M. berdmorei and S. tridecemlineatus (Mercer and Roth 2003), resulting in more highly conserved primers for the former. The 14 microsatellite loci were evaluated in a greater number of individuals: H. phayrei (28 individuals from Mae Rim, Thailand); H. lepidus (eight individuals from Phu Huay Sing, Thailand); and M. berdmorei (28 individuals from Wapipathum, Thailand). PCR reactions were carried out in a DNA Engine thermal cycler (MJ Research) in $2 \mu$ volumes containing $10 \mathrm{ng}$ genomic DNA, $1 \mu \mathrm{l}$ Qiagen Multiplex PCR Master Mix (Qiagen Inc.) and primers $(0.2 \mu \mathrm{M})$. Initial denaturation for $15 \mathrm{~min}$ at $95{ }^{\circ} \mathrm{C}$ was followed by 34 cycles of $30 \mathrm{~s}$ at $94{ }^{\circ} \mathrm{C}, 90 \mathrm{~s}$ at the optimal annealing temperature (Table 1) and $60 \mathrm{~s}$ at $72{ }^{\circ} \mathrm{C}$ with a final extension step of $30 \mathrm{~min}$ at $60^{\circ} \mathrm{C}$. PCR products were run on a 48 -capillary ABI3730 DNA Analyzer using prism set D and a ROX size standard and the alleles sized with GENEMAPPER ver. 3.7 (Applied Biosystems). Observed heterozygosity $\left(H_{O}\right)$, expected heterozygosity $\left(H_{E}\right)$ and estimated null allele frequencies were calculated using CERVUS v3.0.3. Deviations from Hardy-Weinberg equilibrium and tests for linkage 


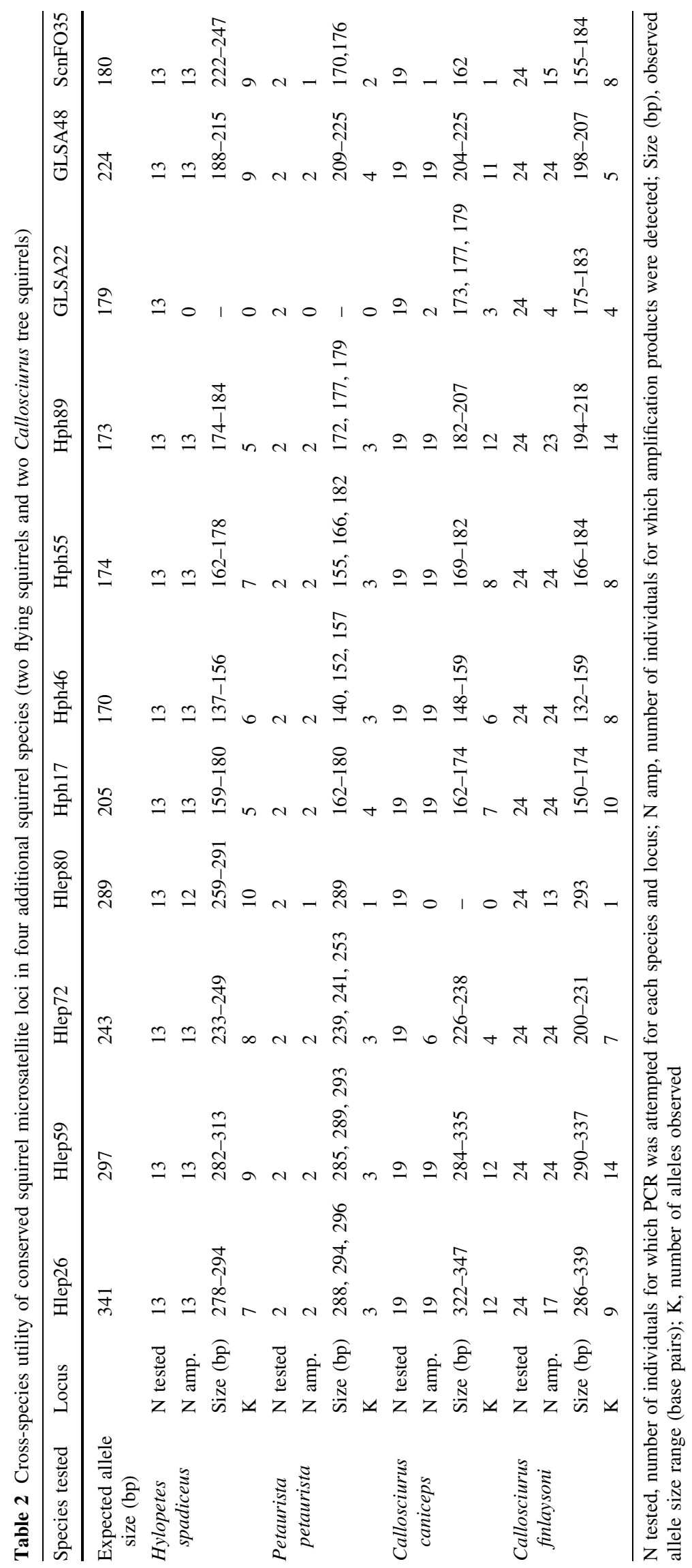


disequilibrium were calculated using GENEPOP v.4.2 (http://genepop.curtin.edu.au/).

Three loci were excluded due to low variability in $M$. berdmorei, one of the three test species (Hph14, Hph54 and Hlep05; Supplementary Table 1). For the remaining 11 loci, the number of alleles per locus across the three species ranged from three to 19 (Table 1). Heterozygotes were observed in males and females for each species suggesting none of the 11 loci were sex-linked. Observed and expected heterozygosities ranged from 0.21 to 0.93 and from 0.23 to 0.96 , respectively (Table 1 ). Two loci deviated from Hardy-Weinberg equilibrium in some species $(\mathrm{p}<0.01$, GLSA22 and Hph46, Table 1), which may be due to null alleles. There was no evidence of linkage disequilibrium between any groups of loci in any species.

The majority of the 11 markers could be amplified and were polymorphic in other squirrel species; ten loci in the flying squirrels Petaurista petaurista and Hylopetes spadiceus and seven loci in the tree squirrels Callosciurus caniceps and $C$. finlaysoni (Table 2). These markers will therefore be useful for conservation studies in a wide range of squirrel taxa.

Acknowledgments We would like to thank Professor Terry Burke for helpful discussions. The microsatellite marker development and genotyping was performed at the NERC Biomolecular Analysis Facility (NBAF) at Sheffield. This study was funded by NBAF grant 658 NBAF-S and the Royal Thai Government.

Open Access This article is distributed under the terms of the Creative Commons Attribution License which permits any use, distribution, and reproduction in any medium, provided the original author(s) and the source are credited.

\section{References}

Armour JAL, Neumann R, Gobert S, Jeffreys AJ (1994) Isolation of human simple repeat loci by hybridization selection. Hum Mol Genet 3:599-605

Dawson DA, Horsburgh GJ, Küpper C et al (2010) New methods to identify conserved microsatellite loci and develop primer sets of high cross-species utility-as demonstrated for birds. Mol Ecol Resour 10:475-494

Mercer JM, Roth VL (2003) The effects of Cenozoic global change on squirrel phylogeny. Science 299(5612):1568-1572

Sodhi NS, Koh LP, Brook BW, Ng PKL (2004) Southeast Asian biodiversity: an impending disaster. Trends Ecol Evol 19(12): 654-660

Sodhi NS, Koh LP, Clements R et al (2010) Conserving Southeast Asian forest biodiversity in human-modified landscapes. Biol Conserv 143(10):2375-2384 